\title{
ABSORÇÃO DE FÓSFORO POR HÍBRIDOS DE MILHO CULTIVADOS EM SOLO DE CERRADO
}

\author{
Cristiano Fernandes ${ }^{1,3} ;$ Takashi Muraoka ${ }^{2,4 *}$ \\ ${ }^{1}$ Fazenda Amazonas - C.P. 13 - CEP: 14230-000 - Serra Azul, SP. \\ ${ }^{2}$ Lab. de Fertilidade do Solo - USP/CENA, C.P. 96 - CEP: 13400-970 - Piracicaba, SP. \\ ${ }^{3}$ Bolsista FAPESP. \\ ${ }^{4}$ Bolsista CNPq. \\ *Autor correspondente<muraoka@cena.usp.br>
}

\begin{abstract}
RESUMO: Nos últimos anos, a região do cerrado tem sido uma das principais áreas de expansão agrícola, porém seus solos apresentam grandes limitações à produção agrícola devido à sua elevada acidez e baixa fertilidade natural, principalmente quanto à disponibilidade de fósforo para as plantas. Este trabalho teve como objetivo comparar 30 híbridos de milho (Zea mays L.), recomendados para a região do cerrado, quando a eficiência na absorção do $\mathrm{P}$ pouco disponível em solos pobres neste nutriente, através da técnica da Diluição Isotópica de ${ }^{32} \mathrm{P}$. Foi utilizado um Latossolo Vermelho-Amarelo distrófico sob duas condições de manejo, um cultivado a 20 anos e outro sob vegetação natural. Houve diferença na absorção de $P$ entre os híbridos avaliados em ambos os solos, sendo que no solo natural o acúmulo de $\mathrm{P}$ e o desenvolvimento das plantas foi bastante reduzido devido à sua baixa fertilidade. Quanto à eficiência na utilização de $P$, houve diferença entre os híbridos, sendo que dos 30 híbridos avaliados, 7 foram classificados como eficientes, 16 como medianamente eficientes e 7 ineficientes, quando plantados no solo cultivado há 20 anos.

Palavras-chave: Zea mays, eficiência a $\mathrm{P}$, fertilidade do solo, diluição isotópica, eficiência nutricional
\end{abstract}

\section{PHOSPHORUS ABSORPTION BY OF CORN HYBRIDS GROWN IN SAVANA SOILS}

\begin{abstract}
In the past years the savana region has been one of the main agricultural expansion areas, however their soils present high limitation for plant growth due their high acidity, low natural fertility, and low phosphorus availability. The objective of this work was to compare 30 of the main recommended maize (Zea mays L.) hybrids for the cerrado region in relation to their ability to absorb poorly-available soil phosphorus through the ${ }^{32} \mathrm{P}$ isotopic dilution technique, using a Typic Dystrarox cultivated for 20 years and another under natural vegetation. Differences in absorption ability were observed among hybrids, seven classified as efficient, sixteen mildly efficient and seven inefficient, for the case of soil cultivated for 20 years. The plant growth and phosphorus concentration in the natural soil was lower due to its low fertility.

Key words: Zea mays, phosphorus efficiency, soil fertility, isotopic dilution, nutritional efficiency
\end{abstract}

\section{INTRODUÇÃO}

Com o grande crescimento populacional, surge a necessidade de aumentar a produção de alimentos no mundo. No Brasil, uma das opções é a expansão da agricultura e o aumento da produtividade nos cerrados; porém nesta região os solos possuem limitações para produção agrícola devido à baixa fertilidade e elevada acidez associada a veranicos e à falta de água disponível na prolongada estação seca. Além disso, um dos principais fatores nutricionais limitantes da produção nestes solos, é a deficiência de fósforo aliada à alta capacidade de fixação deste nutriente.

Solos ácidos e com alta saturação por Al, apresentam problemas na solubilidade de seus compostos, principalmente nutrientes, sendo que, no caso do $\mathrm{P}$, com o abaixamento do $\mathrm{pH}$ ocorre diminuição em sua disponibilidade para as plantas. Outro ponto importante nestes solos é a CTC, ou seja, eles são ricos em sesquióxidos, gibsita e caulinita, cuja quantidade de cargas negativas é baixa resultando em baixa CTC, sendo que em alguns casos, apresenta cargas positivas, aumentando a adsorção de ânions como o ortofosfato. Para minimizar estes problemas, práticas adequadas de manejo do solo, fertilizantes e plantas devem ser adotadas (Goedert et al., 1997). Diversos trabalhos têm sugerido alternativas para solução dos problemas de fertilidade que seriam, além de melhores técnicas de adubação e correção dos solos (Goedert et al., 1985), selecionar cultivares de grãos e pastagens mais tolerantes ao alumínio e mais eficientes na absorção e utilização de fósforo e demais nutrientes (Furlani et al., 1985; Sawazaki \& Furlani, 1987; Macedo, 1996; Bahia Filho et al., 1997).

A capacidade das plantas se desenvolverem em solos com baixo teor de fósforo disponível tem sido atribuída a diversos fatores, incluindo diferenças na morfologia do sistema radicular e densidade dos pelos 
radiculares (Hocking et al., 1997). Assim, o tremoço branco (Lupinus albus) que é uma espécie bem adaptada aos solos ácidos deficientes em P (Gardner et al., 1983) forma raízes proteoides (aglomerado de radículas cobertas com denso emaranhado de pelos radiculares) o que é considerado como sendo resposta a baixa disponibilidade de P (Marschner, 1995). No entanto, Keerthisinghe et al. (1997), constataram que raízes proteoides aparecem também em tremoço branco a concentrações de $P$ normais em solos agrícolas, não sendo essas formações por si só as grandes responsáveis pelo aproveitamento do fósforo. Verificouse que elas excretam grandes quantidades de ácidos cítricos que solubilizam o P "fixado" (Gardner et al., 1983; Keerthisinghe et al., 1997), aumentando a absorção de $\mathrm{P}$ pelas plantas. Da mesma forma, raízes de guandu (Cajanus cajan) excretam ácidos piscídico, malônico e oxálico, o que parece ser o mecanismo pelo qual esta espécie é capaz de liberar o $P$ dos fosfatos de alumínio e férrico (Otani et al., 1996).

Em milho, Alves et al., citados por Parentoni et al. (1999) observaram que genótipos de milho ineficientes para $P$ quando cultivados em solução nutritiva com omissão deste elemento não apresentaram diferenças quanto à morfologia do sistema radicular, enquanto que os considerados eficientes, aumentaram o peso e 0 comprimento das raízes após o estresse de P. Estes aumentos no comprimento foram de $55 \%$ para os híbridos simples, $61 \%$ para o duplo e $112 \%$ para o triplo.

Diversas metodologias podem ser utilizadas para determinação da eficiência de absorção de $P$ pelas plantas, sendo que entre elas está a utilizada por Hocking et al. (1997) na qual compararam a capacidade de diferentes espécies vegetais em acessar $\mathrm{P}$ do solo, através da técnica de diluição isotópica de ${ }^{32} \mathrm{P}$ e cálculo da atividade específica (S). A vantagem dessa técnica é a possibilidade de medir diretamente as diferenças entre plantas na capacidade de absorção de $\mathrm{P}$ menos disponível. Concluíram que o tremoço branco realmente é a espécie mais eficiente, quando comparada com canola, tremoço azul, guandu, soja, girassol e trigo, sendo a soja a menos eficiente.

$A$ atividade específica de ${ }^{32} \mathrm{P}$ é calculada pela relação entre CPM (contagens por minuto) e a quantidade total de $\mathrm{P}$ na planta ( $\mu \mathrm{g}$ de $\mathrm{P}$ ), cuja unidade será CPM $\mu \mathrm{g}^{-1}$, indicando quanto do fósforo presente na planta é proveniente do fertilizante (marcado $c 0 m{ }^{32} \mathrm{P}$ ) e por diferença, quanto é proveniente do solo. Plantas com maior $\mathrm{S}$ absorvem mais $\mathrm{P}$ proveniente do fertilizante que proveniente diretamente do solo, sendo menos eficientes em absorver o nutriente do solo.

Este trabalho teve por objetivo selecionar, entre os híbridos recomendados para a região do cerrado, os mais eficientes na absorção de fósforo em dois solos, um cultivado e adubado há 20 anos e outro sob vegetação natural de cerrado, através da técnica da diluição isotópica de ${ }^{32} \mathrm{P}$.

\section{MATERIAL E MÉTODOS}

Foram utilizados 30 híbridos comerciais de milho recomendados para plantio na região do cerrado e foi conduzido ensaio em casa de vegetação em Piracicaba, SP. Os híbridos selecionados foram: P3071, P30F33, P3081, P30F80, P3027, P3041, P30F45, P3021, P30F88, P30K75, PX1409K, PX1379F, PX1339F, PX1359G, DINA657, DINA766, C813, C909, C747, C333B, ZN8550, ZN8410, ZN8452, ZN8420, ZN8471, AG6018, AG9090, AG7575, DKB911 e TORK, cujas características são apresentadas na Tabela 1. Como cultura padrão, foi utilizado o tremoço branco (Lupinus albus), selecionado através de literatura (Hocking et al., 1997; Fernandes, 2001).

Para a instalação do experimento utilizaram-se duas amostras de Latossolo Vermelho Amarelo distrófico, de textura argilosa, uma proveniente de solo sob cultivo há 20 anos e outra de solo sob vegetação natural, cujas características químicas são as seguintes: a) solo sob vegetação natural: $M O=22,55 \mathrm{~g} \mathrm{~kg}^{-1} ; S$ e $P_{\text {resina }}$ iguais a

Tabela 1 - Principais características dos híbridos estudados.

\begin{tabular}{|c|c|c|c|}
\hline Híbrido & Tipo & Ciclo & Porte \\
\hline P30F80 & Simples & Semi Precoce & Médio \\
\hline C909 & Simples & Precoce & Médio \\
\hline PX1409K & Simples & Semi Precoce & Alto \\
\hline C333B & Simples & Tardio & Médio \\
\hline P30F88 & Simples & Semi Precoce & Médio \\
\hline P3081 & Simples Modificado & Super Precoce & Baixo \\
\hline P3071 & Triplo & Precoce & Médio \\
\hline DINA766 & Simples Modificado & Semi Precoce & Médio \\
\hline P3041 & Triplo & Semi Precoce & Médio \\
\hline P3021 & Triplo & Semi Precoce & Médio \\
\hline ZN8452 & Simples & Semi Precoce & Médio \\
\hline AG7575 & Triplo & Semi Precoce & Médio \\
\hline C747 & Triplo & Semi Precoce & Médio \\
\hline DKB911 & Simples & Precoce & Médio \\
\hline AG6018 & Triplo & Precoce & Médio \\
\hline ZN8550 & Simples & Semi Precoce & Médio \\
\hline PX1379F & Simples & Semi Precoce & Médio \\
\hline TORK & Simples & Semi Precoce & Médio \\
\hline ZN8410 & Simples & Semi Precoce & Médio \\
\hline P30K75 & Simples & Semi Precoce & Baixo \\
\hline AG9090 & * & * & Médio \\
\hline PX1359G & Simples & Semi Precoce & Médio \\
\hline P3027 & Triplo & Semi Precoce & Médio \\
\hline PX1339F & Simples & Precoce & Médio \\
\hline P30F33 & Simples & Precoce & Médio \\
\hline DINA657 & Simples Modificado & Semi Precoce & Médio \\
\hline ZN8471 & Simples & * & Médio \\
\hline C813 & * & * & Médio \\
\hline P30F45 & Simples & Semi Precoce & Alto \\
\hline ZN8420 & Simples & Precoce & Médio \\
\hline
\end{tabular}

${ }^{*}$ Características indeterminadas. 
1,20 e $5,40 \mathrm{mg} \mathrm{dm}^{-3}$, respectivamente, $\mathrm{pH}\left(\mathrm{H}_{2} \mathrm{O}\right) \mathrm{e}$ $\mathrm{pH}\left(\mathrm{CaCl}_{2}\right)$ de 5,02 e 4,12, respectivamente; $\mathrm{K}, \mathrm{Ca}, \mathrm{Mg}$, $\mathrm{H}+\mathrm{Al}$, SB e CTC iguais a $1,60,1,49,1,51,65,81,4,60 \mathrm{e}$ $70,41 \mathrm{mmol}_{\mathrm{c}} \mathrm{dm}^{-3}$, respectivamente; e $\mathrm{V} \%=6,50 \%$; b) solo sob cultivo: $\mathrm{MO}=22,55 \mathrm{~g} \mathrm{~kg}^{-1}$; $\mathrm{S}$ e $\mathrm{P}_{\text {resina }}$ iguais a 2,50 e $26,70 \mathrm{mg} \mathrm{dm}^{-3}$, respectivamente, $\mathrm{pH}\left(\mathrm{H}_{2} \mathrm{O}\right)$ e $\mathrm{pH}\left(\mathrm{CaCl}_{2}\right)$ de 6,11 e 5,32, respectivamente; $\mathrm{K}, \mathrm{Ca}, \mathrm{Mg}$, $\mathrm{H}+\mathrm{Al}$, SB e CTC iguais a $1,80,23,85,9,61,26,05,35,26$ e $61,31 \mathrm{mmol}_{\mathrm{c}} \mathrm{dm}^{-3}$, respectivamente; e V\% $=57,5 \%$. As amostras de solo foram coletadas na camada de $0-20 \mathrm{~cm}$, na Fazenda Alto Alegre, município de Planaltina de Goiás - GO, região do cerrado brasileiro, cuja localização geográfica é: $15^{\circ} 14^{\prime}$ de latitude Sul e $47^{\circ} 42^{\prime}$ de latitude oeste, estando a $826 \mathrm{~m}$ acima do nível do mar.

Para cada tipo de manejo de solo foram feitos 31 tratamentos, ou seja, 30 híbridos de milho e o tremoço branco, cada um com três repetições, totalizando 93 vasos para cada manejo. O esquema experimental utilizado foi o inteiramente casualizado, sendo que os resultados foram submetidos à análise de variância e as médias comparadas pelo teste de Tukey a $5 \%$, dentro de cada tipo de manejo.

Cada vaso, revestido com saco plástico foi preenchido com $1,5 \mathrm{~kg}$ de terra. Aplicou-se a solução de ácido fosfórico marcado, com $6,0 \mathrm{MBq}$ de ${ }^{32} \mathrm{P}$ livre de carregador em copinhos com $20 \mathrm{~g}$ de areia cada um. Após a secagem, homogeneizou-se a areia e adicionouse o conteúdo de cada copinho em um vaso. Homogeneizou-se a terra com a areia e retornou-se ao vaso novamente. Em seguida, irrigaram-se os vasos com $0,5 \mathrm{~L}$ por vaso de água destilada e deixou-se em repouso durante 18 dias com os sacos fechados.

Após o período de repouso, abriram-se os sacos e semearam-se três sementes por vaso de cada híbrido estudado e do tremoço branco, utilizado como planta padrão, sendo três repetições de cada um. A irrigação foi feita quando necessário para manter a umidade do solo aproximadamente em $80 \%$ da capacidade de campo. Passados oito dias do plantio, foi realizado um desbaste deixando duas plantas por vaso. Aos 12 dias após o plantio foi realizada uma adubação com $100 \mathrm{mg}$ $\mathrm{kg}^{-1}$ de $\mathrm{N}$ (sulfato de amônio), $100 \mathrm{mg} \mathrm{kg}^{-1}$ de $\mathrm{K}(\mathrm{KCl})$, $\mathrm{S}$, micronutrientes $(\mathrm{B}, \mathrm{Cu}, \mathrm{Mn}, \mathrm{Zn}$ e Mo) através da aplicação de $10 \mathrm{~mL}$ por vaso da solução de Johanson (Epstein, 1975) e aplicação de $\mathrm{Ca}\left(\mathrm{CaCl}_{2}\right)$ e $\mathrm{Mg}$ $\left(\mathrm{Mg}\left(\mathrm{NO}_{3}\right)_{2}\right)$.

Este ensaio foi conduzido durante 34 dias visto que as plantas dos vasos com solo sob vegetação natural paralisaram seu crescimento devido à baixa fertilidade. Após este período, as plantas foram cortadas e submetidas à secagem em estufa a $60^{\circ} \mathrm{C}$, durante 48 horas. Foram então pesadas para determinação de massa seca e analisadas quimicamente quanto ao teor de $\mathrm{P}$ na parte aérea pelo método de Colorimetria do Metavanadato, descrito por Sarruge \& Haag (1974). A contagem do ${ }^{32} \mathrm{P}$ foi feita por cintilação líquida em Contador de Cintilação em Meio
Líquido, o qual detecta a radiação emitida pelo radioisótopo $\left({ }^{32} \mathrm{P}\right)$ em um determinado período de tempo, obtendo desta forma as contagens por minuto (CPM) para cada amostra. Com os valores do conteúdo de $\mathrm{P}$ e contagem do ${ }^{32} \mathrm{P}$, foi calculada a Atividade Específica (S) para cada híbrido para avaliação da eficiência na absorção do $\mathrm{P}$ proveniente do solo. A atividade específica foi calculada dividindo-se as contagens de ${ }^{32} \mathrm{P}$ nas plantas de cada vaso pela respectiva quantidade de $P$ acumulada nas plantas. Quanto menor a atividade específica, maior a eficiência na absorção de $\mathrm{P}$ do solo e vice-versa.

\section{RESULTADOS E DISCUSSÃO}

Durante a condução do experimento foram observados alguns problemas nas plantas, decorrentes da baixa fertilidade dos solos utilizados. No solo sob vegetação natural, aos 12 dias após a germinação foi visualizado que na maioria dos vasos havia raízes aflorando na superfície do solo, embora o sistema radicular não tenha sido avaliado, resultado da elevada acidez e da sensibilidade das plantas. Aos 18 dias a maioria dos híbridos já apresentava sintomas de deficiência de $P$, arroxeamento de folhas velhas.

Durante todo o ensaio os híbridos cultivados no solo sob vegetação natural apresentaram reduzido crescimento e permaneceram raquíticos, com grandes problemas de falta de fósforo e deficiências nutricionais generalizadas. Assim, os resultados obtidos para o solo sob vegetação natural devem ser considerados com ressalvas, uma vez que somente híbridos mais rústicos apresentaram desenvolvimento ligeiramente melhor, ou seja, possuem características que favorecem a obtenção dos nutrientes necessários ao desenvolvimento, mesmo em condições de baixa disponibilidade, como é o caso do fósforo em solo de cerrado. Estas características estão relacionadas com diferenças morfológicas no sistema radicular, liberação de ácidos que solubilizam o $P$ "fixado" e ainda plantas metabolicamente adaptadas que necessitam de menor teor de nutriente disponível para um bom desenvolvimento. Já no solo cultivado, houve variação no desenvolvimento das plantas, assim como alguns híbridos apresentaram sintomas claros de deficiência de $\mathrm{P}$, porém menos intensos que no solo sob vegetação natural.

Houve diferença na produção de matéria seca entre os híbridos estudados em ambos os solos (Tabelas 2 e 3). No solo sob vegetação natural, devido à quantidade muito pequena de fósforo disponível ( $P$ resina $=5,4 \mathrm{mg} \mathrm{dm}^{-3}$ ) as plantas se desenvolveram pouco, sendo a produção de matéria seca da parte aérea duas a quatro vezes menor do que no solo cultivado há 20 anos, para todos os híbridos, com uma média de produção de 1,08 g por vaso (Tabela 3). Os híbridos ZN8471, ZN8410, entre outros, e o tremoço branco, por estarem mais bem adaptados às condições restritivas do 
Tabela 2 - Produção de matéria seca (MS), teor e acúmulo de P na parte aérea e atividade específica (S) em híbridos de milho e tremoço branco avaliados em solo de cerrado "cultivado".

\begin{tabular}{|c|c|c|c|c|}
\hline Hibrido & MS & $\mathrm{P}$ & $\mathrm{P}$ acumulado & $S$ \\
\hline & $\mathrm{g}$ & $\mathrm{g} \mathrm{kg}^{-1}$ & $\mathrm{mg}$ & CРM $\mu \mathrm{g}^{-1}$ \\
\hline P30F80 & 3,52 abcdef & $2,13 \mathrm{abcd}$ & $7,54 \mathrm{bcd}$ & $45,63 \mathrm{a}$ \\
\hline C909 & 3,41 abcdef & $2,22 a b$ & $7,57 \mathrm{bcd}$ & $44,37 \mathrm{ab}$ \\
\hline PX1409K & 3,91 abcde & $2,13 \mathrm{abcd}$ & $8,30 a b$ & $43,85 a b c$ \\
\hline C333B & 3,92 abcde & $1,95 \mathrm{abcd}$ & $7,65 \mathrm{bcd}$ & $43,77 \mathrm{abc}$ \\
\hline P30F88 & $4,04 \mathrm{abcd}$ & $2,03 \mathrm{abcd}$ & $8,22 a b$ & $43,62 a b c$ \\
\hline P3081 & 3,89 abcde & 1,96 abcd & $7,63 \mathrm{bcd}$ & $43,55 \mathrm{abc}$ \\
\hline P3071 & 3,46 abcdef & $2,23 a b$ & 7,72 bcd & $43,34 \mathrm{abcd}$ \\
\hline DINA766 & 2,92 defgh & $2,24 a b$ & $6,53 \mathrm{bcdef}$ & 41,72 abcde \\
\hline P3041 & 3,76 abcdef & 2,07 abcd & 7,74 bcd & 41,57 abcde \\
\hline P3021 & $4,49 a b$ & $1,64 \mathrm{~d}$ & 7,33 bcde & 41,55 abcde \\
\hline ZN8452 & $4,34 a b c$ & $2,41 \mathrm{a}$ & $10,49 a$ & 41,42 abcde \\
\hline AG7575 & 3,83 abcdef & 2,10 abcd & $8,01 a b$ & 41,18 abcdef \\
\hline C747 & 3,70 abcdef & $1,94 \mathrm{abcd}$ & 7,05 bcdef & 40,50 abcdef \\
\hline DKB911 & 4,09 abcd & $1,86 \mathrm{bcd}$ & 7,61 bcd & 40,49 abcdef \\
\hline AG6018 & 3,76 abcdef & 2,09 abcd & 7,88 abcd & 40,27 abcdef \\
\hline ZN8550 & $4,56 \mathrm{a}$ & $1,73 \mathrm{bcd}$ & $7,90 \mathrm{abc}$ & 40,14 abcdef \\
\hline PX1379F & 3,96 abcde & 1,72 bcd & 6,82 bcefg & 39,79 abcdef \\
\hline TORK & $2,04 \mathrm{gh}$ & $1,88 \mathrm{abcd}$ & $3,84 \mathrm{ghi}$ & 39,65 abcdef \\
\hline ZN8410 & 3,86 abcde & $2,17 a b c$ & $8,24 a b$ & 39,26 abcdefg \\
\hline P30K75 & $4,40 a b$ & $1,71 \mathrm{bcd}$ & $7,52 \mathrm{bcd}$ & 38,69 bcdefg \\
\hline AG9090 & $1,71 \mathrm{~h}$ & 1,97 abcd & $3,37 \mathrm{ghi}$ & 38,64 bcdefg \\
\hline$P \times 1359 G$ & 3,22 bcdefg & $1,65 \mathrm{~cd}$ & 5,30 cdefgh & 37,42 cdefg \\
\hline P3027 & 2,65 efgh & $1,81 \mathrm{bcd}$ & 4,77 efghi & 36,62 defg \\
\hline PX1339F & $2,50 \mathrm{fgh}$ & $1,78 \mathrm{bcd}$ & $4,48 \mathrm{fghi}$ & 35,95 efg \\
\hline P30F33 & 3,47 abcdef & $2,00 \mathrm{abcd}$ & 6,95 cdef & 35,78 efg \\
\hline DINA657 & 3,26 abcdefg & 2,10 abcd & 6,90 bcdef & 35,17 efg \\
\hline ZN8471 & 4,05 abcd & $1,81 \mathrm{bcd}$ & 7,31 bcde & 34,91 efg \\
\hline C813 & $1,88 \mathrm{~h}$ & $1,61 \mathrm{~d}$ & $3,04 \mathrm{hi}$ & 34,81 efg \\
\hline P30F45 & 2,90 defgh & 1,79 bcd & 5,23 defgh & $34,45 \mathrm{fg}$ \\
\hline ZN8420 & 3,02 cdefgh & $1,97 \mathrm{abcd}$ & 5,98 bcdefg & $32,40 \mathrm{~g}$ \\
\hline Tremoço & $2,52 \mathrm{fgh}$ & $0,99 \mathrm{e}$ & $2,48 \mathrm{i}$ & $13,89 \mathrm{~h}$ \\
\hline $\mathrm{F}$ & $10,13^{*}$ & $7,69^{*}$ & $14,41^{*}$ & $21,56^{*}$ \\
\hline CV (\%) & 11,99 & 8,63 & 12,31 & 5,52 \\
\hline Média & 3,45 & 1,93 & 6,69 & 38,85 \\
\hline
\end{tabular}

*Médias seguidas pelas mesmas letras na coluna não diferem pelo teste de Tukey a $5 \%$.

solo, foram os que produziram mais matéria seca na parte aérea, enquanto o TORK, C813 e AG9090 foram os que menos produziram quando plantados no solo sob vegetação natural (Tabela 3), apresentando menor capacidade de obtenção de nutrientes nestas condições.

No solo cultivado, houve grande variação na produção de matéria seca entre os híbridos (Tabela 2), variando de 1,71 a $4,56 \mathrm{~g}$, sendo que os híbridos ZN8550, P3021 e P30K75 foram os mais produtivos, produzindo $4,56,4,49$ e $4,40 \mathrm{~g}$, respectivamente, enquanto os híbridos AG9090, C813 e TORK foram os menos produtivos, produzindo 1,71, 1,88 e 2,04 g respectivamente.
Há grande variação na matéria seca produzida por híbridos de milho quando cultivados em solos pobres em $\mathrm{P}$ disponível. Esta variação está relacionada com a capacidade de absorção e utilização de nutrientes pelos híbridos, porém, isso não indica que são mais ou menos eficientes na absorção do $\mathrm{P}$ do solo. Esta produção de parte aérea é resultado de uma associação de fatores, como maior capacidade de absorção de nutrientes, menor exigência de nutrientes disponível no solo, entre outros. Quanto aos teores de P na planta (Tabela 2), também houve grande variação entre os híbridos, independentemente das características morfológicas, nos dois solos utilizados, porém, os resultados do solo sob 
vegetação natural não serão comentados devido à pequena quantidade de $\mathrm{P}$ disponível para as plantas, as quais apresentaram desenvolvimento reduzido, possivelmente pela falta de nutrientes, inviabilizando assim a comparação entre os híbridos. Neste solo, pode ter ocorrido o efeito da diluição, ou seja, a disponibilidade de nutrientes neste solo é baixa, e a falta de outros nutrientes pode ter afetado o desenvolvimento da planta, que pode apresentar maior teor de $\mathrm{P}$ do que uma planta normal. Entretanto, a atividade específica (S) compara diretamente a relação ${ }^{32} \mathrm{P} /{ }^{31} \mathrm{P}$, que é inversamente proporcional à capacidade de absorção da planta.

No solo sob cultivo, o teor médio de $P$ na planta foi de $1,93 \mathrm{~g} \mathrm{~kg}^{-1}$, sendo que a variação foi de $0,93 \mathrm{~g} \mathrm{~kg}^{-1}$ para o tremoço branco a 2,41 $\mathrm{g} \mathrm{kg}^{-1}$ para o híbrido ZN8452. Já o acúmulo médio de $P$ pelos híbridos foi de $9,78 \mathrm{mg}$ por vaso, onde os híbridos ZN 8452, PX1409K, ZN 8410, P 30F88, AG 7575, ZN 8550 e AG 6018 foram os que mais acumularam $P$ na parte aérea. Os híbridos C813, AG 9090, TORK, PX1339F, P3027 e o tremoço foram os que menos acumularam $P$ (Tabela 2). Este acúmulo de $P$ não indica que as plantas são mais ou menos eficientes, uma vez que híbridos através de adaptações genéticas podem se desenvolver e produzir bem com menor quantidade de $\mathrm{P}$, ou seja, plantas com alta quantidade de $\mathrm{P}$ podem não ser mais produtivas e vice-versa (Machado, 2000), assim como alguns híbridos podem apresentar diferença na eficiência de utilização do fósforo absorvido, necessitando de menos nutriente absorvido para um melhor desenvolvimento. Baker et al. (1970) e Machado et al. (1999) também mostraram haver diferenças geneticamente controladas no acúmulo de $\mathrm{P}$ por híbridos de milho, mas que este acúmulo não estava relacionado com a habilidade das plantas em extrair $\mathrm{P}$ do solo, podendo ser resultado do efeito da diluição.

Para selecionar os híbridos mais eficientes na absorção de $\mathrm{P}$ do solo, foram calculadas as atividades específicas de ${ }^{32} \mathrm{P}$ para cada um em ambos os solos, sendo que as plantas cultivadas no solo sob vegetação natural apresentaram desenvolvimento reduzido devido à baixa quantidade de $\mathrm{P}$ total no solo $\left(225,7 \mathrm{mg} \mathrm{dm}^{-3}\right)$, grande parte deve estar fortemente adsorvido aos colóides e conseqüentemente muito pouco disponível para as plantas, onde nenhum híbrido pode ter sido capaz de extrair o P do solo.

Se todos os híbridos fossem idênticos quanto à eficiência na absorção de $P$, e cultivados no mesmo solo sob as mesmas condições, eles deveriam apresentar a mesma atividade específica, ou seja, a relação CPM $\mu \mathrm{g}^{-1}$ de $P$ presente na planta seria igual. Mas vários trabalhos mostram diferenças adaptativas entre espécies de plantas (Rorison, 1968; Furlani et al., 1985; Sawazaki \& Furlani, 1987; Macedo, 1996; Bahia Filho et al., 1997; Hocking et al., 1997) e mesmo entre genótipos de milho (Anghinoni et al., 1989; Baligar et al., 1997; Machado et al., 1999; Parentoni et al., 1999; Machado, 2000), logo, a atividade específica de ${ }^{32} \mathrm{P}$ deve diferir entre os híbridos, como ocorreu no presente trabalho.
A atividade especifica média foi de 38,85 CPM $\mu \mathrm{g}^{-1}$, sendo que houve diferença entre os híbridos estudados (Tabela 2 e Figura 1). Os híbridos P30F80 e C909 foram os que apresentaram maior atividade específica, 45,63 e 44,37 CPM $\mu^{-1}$, respectivamente, sendo considerados ineficientes segundo Hocking et al. (1997), enquanto o ZN8420, P30F45, C813, ZN8471, DINA657, P30F33 e PX1339F foram mais eficientes na absorção do $\mathrm{P}$ do solo, cujos valores de atividade específica situam na faixa de 32,40 a $35,80 \mathrm{CPM} \mu \mathrm{gg}^{-1}$. Estas diferenças na eficiência a $P$ podem ser herdadas geneticamente através do cruzamento de linhagens capazes de produzir híbridos mais eficientes em condições de estresse de fósforo, como observado por Parentoni et al. (1999) quando avaliou híbridos simples obtidos de linhagens eficientes.

O tremoço branco, considerado por Hocking et al. (1997) como cultura mais eficiente na absorção de P pouco disponível do solo, e utilizado neste experimento como cultura de referência, também foi mais eficiente que todos os híbridos estudados, apresentando uma atividade especifica 2,3 vezes menor que a do melhor híbrido, cujo valor foi de 13,89 CPM $\mu^{-1}$.

Os híbridos avaliados foram divididos arbitrariamente em três classes quanto a sua eficiência na absorção de fósforo. Ineficientes, cuja atividade

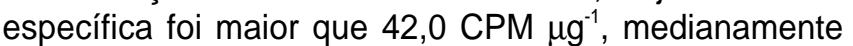
eficientes, com valores entre 36,0 e 42,0 CPM $\mu \mathrm{g}^{-1}$, e eficientes, cuja atividade especifica foi menor que 36,0 CPM $\mu \mathrm{g}^{-1}$, como ilustrado na figura 1.

Dentre os híbridos atualmente recomendados para a região do cerrado, o ZN8420, P30F45, C813, ZN8471, DINA657, P30F33 e PX1339F, todos simples, exceto o DINA657 (simples modificado), sendo mais eficientes na absorção do fósforo pouco disponível do solo, além da característica de precocidade também favorável para a região, devem ser avaliados quando a produtividade de grãos em campo. Em um experimento de campo no mesmo solo utilizado neste experimento, Fernandes (2001) observou que o híbrido P30F33,

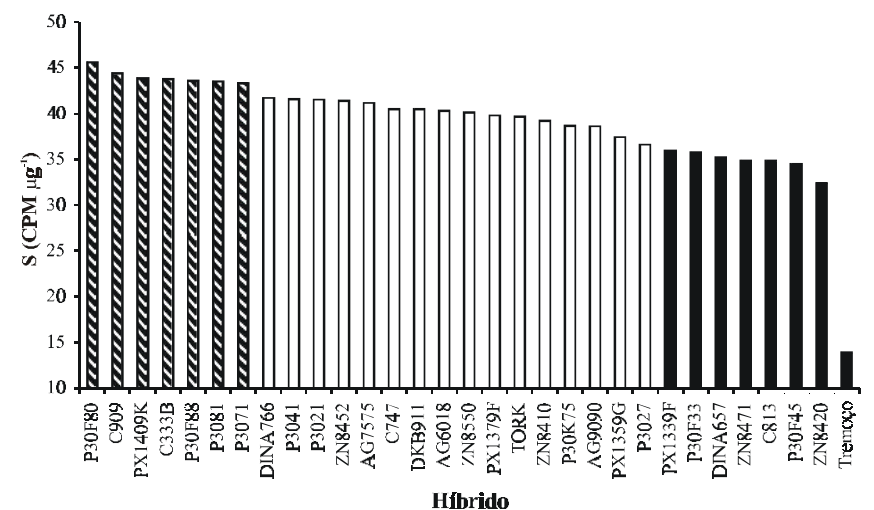

Figura 1 - Classificação dos híbridos como: $\mathbb{Z}$ Ineficiente, $\square$ Medianamente eficiente e Eficiente na absorção de $\mathrm{P}$, de acordo com a atividade específica $\mathrm{S}\left(\mathrm{CPM} \mu \mathrm{g}^{-1}\right)$. 
quando comparado com outros 27 híbridos de milho, está entre os mais produtivos, com rendimentos superiores a 9,0 t ha ${ }^{-1}$. Para os híbridos estudados, o ciclo não influenciou na eficiência na absorção de fósforo proveniente do solo.

Houve diferença entre as atividades específicas dos híbridos no solo sob vegetação natural (Tabela 3), sendo que a atividade média foi de 2,91 CPM $\mu \mathrm{g}^{-1}$, muito

Tabela 3 - Atividade específica (S) e matéria seca (MS) da parte aérea de híbridos avaliados em solo de cerrado sob vegetação natural.

\begin{tabular}{|c|c|c|}
\hline Hibrido & $S$ & MS \\
\hline & $\mathrm{CPM} \mu \mathrm{g}^{-1}$ & $g$ \\
\hline P30F80 & $1,98 \mathrm{~g}$ & 0,903 efghij \\
\hline C909 & $3,29 \mathrm{cdef}$ & 0,797 hijk \\
\hline PX1409K & $2,29 \mathrm{~g}$ & $1,263 \mathrm{abcd}$ \\
\hline С33зВ & 3,43 bcde & 0,873 fghij \\
\hline P30F88 & $2,05 \mathrm{~g}$ & 1,123 bcdefg \\
\hline P3081 & $2,41 \mathrm{fg}$ & 1,120 bcdefg \\
\hline P3071 & $2,31 \mathrm{~g}$ & 0,920 efghij \\
\hline DINA766 & $3,58 \mathrm{bcd}$ & 0,820 ghijk \\
\hline P3041 & 3,34 cdef & 0,967 defghi \\
\hline P3021 & $2,53 \mathrm{efg}$ & $1,330 \mathrm{abc}$ \\
\hline ZN8452 & $2,12 \mathrm{~g}$ & $1,310 \mathrm{abc}$ \\
\hline AG7575 & 2,84 defg & 1,143 bcdef \\
\hline C747 & $2,26 \mathrm{~g}$ & 0,867 fghij \\
\hline DKB911 & 3,48 bcde & 1,040 cdefgh \\
\hline AG6018 & 2,58 efg & 1,163 bcdef \\
\hline ZN8550 & 2,96 cdefg & 1,163 bcdef \\
\hline PX1379F & 2,70 defg & 1,207 bcde \\
\hline TORK & $3,88 b c$ & $0,717 \mathrm{ijk}$ \\
\hline ZN8410 & $2,20 \mathrm{~g}$ & $1,400 a b$ \\
\hline P30K75 & 2,53 efg & 1,203 bcde \\
\hline AG9090 & $6,12 \mathrm{a}$ & $0,517 \mathrm{k}$ \\
\hline PX1359G & 2,54 efg & 1,140 bcdef \\
\hline P3027 & $3,85 b c$ & 1,033 cdefghi \\
\hline PX1339F & $2,30 \mathrm{~g}$ & 1,177 bcdef \\
\hline P30F33 & $2,22 \mathrm{~g}$ & $1,253 \mathrm{abcd}$ \\
\hline D INA657 & 2,77 defg & 1,147 bcdef \\
\hline ZN8471 & $1,98 \mathrm{~g}$ & $1,427 a b$ \\
\hline C813 & $4,39 \mathrm{~b}$ & $0,633 \mathrm{jk}$ \\
\hline P30F45 & 3,50 bcde & 0,967 defghi \\
\hline ZN8420 & 3,44 bcde & 1,173 bcdef \\
\hline Tremoço & $2,45 \mathrm{fg}$ & $1,530 \mathrm{a}$ \\
\hline $\mathrm{F}$ & $24,90^{*}$ & $16,82^{*}$ \\
\hline CV (\%) & 10,44 & 9,22 \\
\hline Média & 2,91 & 1,08 \\
\hline
\end{tabular}

*Médias seguidas pelas mesmas letras na coluna não diferem pelo teste de Tukey a $5 \%$. inferior à média no solo cultivado, além do menor acúmulo de $\mathrm{P}$ na parte aérea das plantas cultivadas no solo sob vegetação natural $\left(0,91 \mathrm{~g} \mathrm{~kg}^{-1}\right)$. $\mathrm{O}$ resultado deste baixo acúmulo de $\mathrm{P}$ e baixa atividade específica (S) é devido ao reduzido teor de fósforo no solo e à alta fixação do ${ }^{32} \mathrm{P}$ aplicado. Neste solo, os híbridos com $\mathrm{S}$ menor que 2,4 CPM $\mu^{-1}$, arbitrariamente, são considerados eficientes na absorção de fósforo do solo, apesar do pequeno crescimento (Tabela 3). Já os híbridos com $\mathrm{S}$ maior que 3,4 $\mathrm{CPM} \mu \mathrm{g}^{-1}$ são ineficientes, enquanto os demais são considerados medianamente eficientes, lembrando que estes resultados devem ser considerados com ressalvas, pois as plantas podem não ter expressado seu potencial na absorção de $\mathrm{P}$ devido a grande fixação pelo solo e baixa fertilidade, prejudicando seu desenvolvimento.

\section{AGRADECIMENTOS}

Ao Eng. Agrônomo Edemar Joaquim Corazza pelo auxílio nas correções do trabalho e na seleção dos híbridos e à FAPESP e IAEA (Projeto RC-BRA-10954) pelo apoio financeiro.

\section{REFERÊNCIAS BIBLIOGRÁFICAS}

ANGHINONI, I.; VOLKART, C.R.; FATTORE, N.; ERNANI, P.R. Morfologia de raízes e cinética da absorção de nutrientes em diversas espécies e genótipos de plantas. Revista Brasileira de Ciência do Solo, v.13, p.355361, 1989.

BAHIA FILHO, A.F.C.; MAGNAVACA, R.; SCHAFFERT, R.E.; ALVES, V.M.C. Identification, utilization, and economic impact of maize germplasm tolerant to low levels of phosphorus and toxic levels of exchangeable aluminum in Brasilian soils. In: MONIZ, A.C.; FULANI, A.M.C.; SCHAFFERT, R.E.; FAGERIA, N.K.; ROSOLEM, C.A.; CANTARELLA; H. Plant-soil interactions at low $\mathrm{pH}$ : Sustainable agriculture and forestry production. Brasília: SBCS, 1997. p.59-70.

BAKER, D.E.; JARRE, A.E.; MARSHALL, L.E.; THOMAS, N.I. Phosphorus uptake from soils by corn hybrids selected for high and low phosphorus accumulation. Agronomy Journal, v.62, p.103-106, 1970.

BALIGAR, V.C.; PITTA, G.V.E.; GAMA, E.E.G.; SCHAFFERT, R.E.; BAHIA FILHO, A.F.C.; CLARK, R.B. Soil acidity effects on nutrient use efficiency in exotic maize genotypes. Plant and Soil, v.192, p.9-13, 1997.

FERNANDES, C. Eficiências de diferentes culturas e híbridos de milho quanto à utilização de fósforo em solos de cerrado. Piracicaba, 2001. 51p. Dissertação (Mestrado) - Escola Superior de Agricultura "Luiz de Queiroz", Universidade de São Paulo.

EPSTEIN, E. Nutrição mineral de plantas: princípios e perspectivas. Rio de Janeiro: Livros Técnicos e Científicos, 1975. 341p.

FURLANI, A.M.C.; BATAGLIA, O.C.; LIMA, M. Eficiência de linhagens de milho na absorção e utilização de fósforo em solução nutritiva. Bragantia, v.44, p.129-147, 1985.

GARDNER, W.K.; BARBER, D.A.; PARBERRY, D.G. The acquisition of phosphorus by Lupinus albus L. III. The probable mechanism by which phosphorus movement in the soil/root interface is enhanced. Plant and Soil, v.70, p.107-124, 1983.

GOEDERT, W.J.; LOBATO, E.; LOURENÇO, S. Nutrient use efficiency in brasilian acid soils: Nutrient management and plant efficiency. In: MONIZ, A.C.; FULANI, A.M.C.; SCHAFFERT, R.E.; FAGERIA, N.K.; ROSOLEM, C.A.; CANTARELLA; H. Plant-soil interactions at low pH: Sustainable agriculture and forestry production. Brasília: SBCS, 1997. p.97-104.

GOEDERT, W.J.; SOUZA, D.M.G.; LOBATO, E. Fósforo. In: GOEDERT, W.J. Solos dos cerrados. Tecnologias e estratégias de manejo. São Paulo: Nobel; EMBRAPA, 1985. p.129-166.

HOCKING, P.J.; KEERTHISINGHE, G.; SMITH, F.W.; RANDALL, P.J. Comparation of the ability of different crop species to access poorlyavailable soil phosphorus. In: ANDO, T.; FUGITA, K.; MAE, T.; MATSUMOTO, H.; MORI, S.; SEKIYA, J. Plant nutrition - for sustainable food production and environment. Dordrecht: Kluwer Academic Publishers, 1997. p.305-308. 
KEERTHISINGHE, G.; HOCKING, P.J.; RYAN, P.R.; DELHAIZE, E. Effect of phosphorus supply on the formation and function of proteoid roots of white lupin (Lupinus albus L.) Plant, Cell and Environment, v.21, p.467-478, 1997.

MACEDO, J. Os solos da região dos cerrados. In: ALVAREZ, V.H.V. O solo nos grandes domínios morfoclimáticos do Brasil e o desenvolvimento sustentado. Viçosa: SBCS; UFV, 1996. p.135-155.

MACHADO, C.T.T. Caracterização de genótipos de milho quanto a parâmetros morfológicos, fisiológicos e microbiológicos associados à eficiência de absorção e uso de fósforo. Rio de Janeiro, 2000. 366p. Tese (Doutorado) - Universidade Federal Rural do Rio de Janeiro.

MACHADO, C.T.T.; GUERRA, J.G.M.; ALMEIDA, D.L.; MACHADO, A.T. Variabilidade entre genótipos de milho para eficiência no uso de fósforo. Bragantia, v.58, p.109-124, 1999.

MARSCHNER, H. Mineral nutrition of higher plants. 2.ed. London: Academic Press, 1995. 889p.

OTANI, T.; AE, N.; TANAKA, M. Phosphorus uptake mechanisms of crop growth in soils with low P status. Soil Science and Plant Nutrition, v.42, p.553-560, 1996.
PARENTONI, S.N.; GAMA, E.E.G.; SANTOS, M.X.; LOPES, M.A.; ALVES V.M.C.; BAHIA FILHO, A.F.C.; VASCONCELLOS, C.A.; MAGNAVACA, R.; PACHECO, C.A.P.; MEIRELLES, W.F.; GUIMARÃES, P.E.O.; SILVA, A.E.; NASPOLINI FILHO, W.; MORO, J.R.; VIANA, R.T.; PITTA, G.V.E.; FRANCA, G.E.; PURCINO, A.A.C.; SOUZA, I.R.P.; ARRIEL, I.E MACHADO, A.T.; CORREA, L.A.; OLIVEIRA, A.C.; PAIVA, E. Adaptação de milho a solos ácidos: Tolerância à toxidez de alumínio e eficiência no uso de nutrientes no programa de pesquisa da EMBRAPA - Milho e Sorgo. In: REUNION LATINOAMERICANA DEL MAIZ, 18., Sete Lagoas Memórias. Sete Lagoas: EMBRAPA -Milho e Sorgo; México: CIMMYT, 1999. p.179-199.

RORISON, I.H. The response to phosphorus of some ecologically distinct plant species. I. Growth rates and phosphorus absorption. New Phytologist, v.67, p.913-923, 1968.

SARRUGE, J.R.; HAAG, H.P. Análises químicas em plantas. Piracicaba: ESALQ, Departamento de Química, 1974. 56p.

SAWAZAKI, E.; FURLANI, P.R. Genética da tolerância ao alumínio em milho cateto. Bragantia, v.46, p.269-278, 1987.

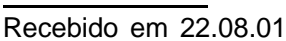

